\title{
Documentos en náhuatl en Cracovia
}

\author{
Brígida von Mentz
}

$\mathrm{L}$ a antigua ciudad de Cracovia a orillas del río Vístula en el sur de Polonia, desde la Edad Media cuenta con una de las universidades más importantes de Europa. En la famosa Biblioteca Jagiellowska de esta casa de estudios se alberga valiosa documentación histórica. A esta biblioteca han llegado varios manuscritos en náhuatl. Como recientemente tuve oportunidad de revisarlos quisiera describirlos en este ensayo y llamar la atención sobre su importancia.

Se trata de un corpus de documentos coloniales iberoamericanos escritos en diversas lenguas como náhuatl, tarasco, guaraní y castellano que tienen las siglas "Am." [de Americana] con los números 3, 4, 6, 8, 9, 11 hasta 15, incluyendo unas pinturas de Venezuela fechadas en 1840. Formaban parte de las adquisiciones que realizó la casa reinante de Prusia en el siglo XIX y que se resguardaron en la Biblioteca Real de Berlín, la que hoy se conoce como Staatsbibliothek [Biblioteca Estatal], con dos sedes, una en la parte centro-occidental de la ciudad y otra en el antiguo centro histórico.

Durante la Segunda Guerra Mundial estos manuscritos fueron trasladados fuera de Berlín y se perdieron en el caos de la conflagración militar. Finalmente, gracias a la labor de los historiadores en Polonia fueron reunidos todos los documentos procedentes de Berlín y otros lugares en la Biblioteca Jagiellowska. También se encuentran entre ellos textos en alemán que forman parte de legados documentales de importantes poetas o personajes públicos, como parte de la testamentaría de los hermanos Alejandro y Guillermo de Humboldt. Entre los documentos relativos a México también hay ediciones facsimilares de los códices Dresden, Pérez, Troano, Mayo y Nuttal de fines del siglos XIX o del XX con la clasificación Ms. Zs. 14590, 14 620, 14 623, 14652 y 16 442, respectivamente.

Los documentos de la colección "Americana" de Berlín que ahora están en Cracovia son los siguientes: un fragmento en náhuatl de censo denominado "Eine Aztekische Handschrift" [Un manuscrito azteca] (Am. 3), del que hablaremos con mayor detalle más abajo; los "Títulos de Santa Isabel Tola", manuscrito en español y náhuatl (Am. 4); "Pinturas de Venezuela", de 1840 (Am. 6); un "Fragmento de una lista tributaria en idioma mexicano" (Am. 8), que también analizaremos con mayor cuidado en este ensayo; "Un documento jurídico de un proceso, en castellano" (Am. 9); un "Facsímil publicado por Paso y

BRÍGIDA VON MENTZ: CIESAS.

Desacatos, núm. 12, otoño 2003, pp. 163-17o. 




Códice Am.3 de Cracovia (detalle).

Troncoso" (Am. 11); "Dos documentos en lengua guaraní" (Am. 12 y 13), y "Sermones en tarasco" (Am. 14 y 15). ${ }^{1}$

La revisión de los manuscritos mostró que el censo en náhuatl registrado con las siglas Am. 3 que consta de 75 fojas, se puede completar con el fragmento catalogado como Am. 8, pues este último consiste en dos fojas del mismo censo. El primero fue donado por un comerciante de apellido Hermann al rey de Prusia en 1834. Como

\footnotetext{
${ }^{1}$ Mayor información sobre estos documentos se encuentra en Ulf Bankmann, "Manuscripta Americana der Staatsbibliothek in Berlin", en Verhandlungen des XXXVIII Internationales Amerikanistenkongresses, Stuttgart-Muenchen, 12-18 de agosto de 1968, t. II, Munich, Alemania, 1970.
}

consta en el sello rojo "Ex Biblioth. Regis Berlinensis" y en sus etiquetas, fue catalogado primero como la adquisición 484 en junio de 1834, época en la que fungía como bibliotecario Eduard Buschmann, quien había visitado México, era conocedor del náhuatl y tuvo una gran cercanía con los hermanos Humboldt, ambos interesados intensamente en las culturas americanas.

Guillermo de Humboldt se hacía preguntas sobre la diversidad, origen y desarrollo de las lenguas e incluso, en la década de 1820, redactó una gramática del náhuatl. Para realizar sus estudios se apoyaba en documentos e información que le proporcionaba su hermano y el propio Buschmann. Las inquietudes académicas de estos personajes y sus intereses lingüísticos, históricos y de índole naturalista explican, por lo tanto, el interés que en la primera mitad del siglo XIX se tenía en Berlín por adquirir pictografías, textos en lenguas indígenas y objetos arqueológicos relacionados con los "antiguos mexicanos".

Los documentos en náhuatl Am. 3 comprados en 1834 y posteriormente ampliados con una compra adicional en 1862 de dos fojas más, clasificados como Am. 8, ubicados hoy en día en Cracovia, están escritos en papel amate de 42 por $25 \mathrm{~cm}$. Corresponden con exactitud, a su vez, a los censos escritos en el mismo papel y con la misma grafía y tinta que se encuentran en la Biblioteca $\mathrm{Na}$ cional de Francia (BNF) en París con la denominación manuscrito mexicain $393 .{ }^{2}$ Todos estos textos son especialmente atractivos académicamente para los interesados en la lengua náhuatl o en la historia social de la región de Morelos hacia 1540, pues se trata de tres fragmentos más de los llamados "Censos de Morelos" y que están ubicados dos en Cracovia y uno en París. ${ }^{3}$ Los otros están en la ciudad de México bajo resguardo del INAH (en la Biblioteca del Museo Nacional de Antropología e Historia) y conforman las partes más amplias. ${ }^{4}$

\footnotetext{
${ }^{2}$ En el proyecto Amoxcalli del CIESAS se prepara actualmente la publicación de ese fragmento.

${ }^{3}$ Ambos documentos están en proceso de traducción y edición por quien esto suscribe. Además se realiza un estudio de historia social de la zona basado sobre todo en esos materiales.

${ }^{4}$ Ms. 549, 550 y 551, colección antigua, Archivo Histórico, en la Biblioteca del Museo Nacional de Antropología, INAH. En las décadas de 1960 y 1970 Pedro Carrasco había mencionado la importancia de
} 
Estos censos en náhuatl de época tan temprana fueron elaborados aproximadamente entre 1535 y 1544 y probablemente sirvieron para documentar judicialmente el número de vasallos y de tributos del marqués del Valle de Oaxaca, Hernán Cortés. Éste se enfrentaba en esa época a numerosos rivales y se le acusaba de que el número de vasallos indios y las cantidades de tributos que les exigía eran excesivos y sobrepasaban sus prerrogativas y derechos obtenidos en 1529. Estos padrones o censos que los señores indígenas elaboraban con regularidad cotidiana (probablemente desde la época anterior a la conquista para tener control de la población, del uso del suelo y de las contribuciones) conforman una fuente extremadamente rica y valiosa tanto para la demografía histórica, el conocimiento del parentesco y la estratificación social indígena, así como para el estudio del tributo y la economía regional. Arrojan luz incluso sobre la forma de organización interna de las pueblos mayores así como de comunidades pequeñas y minúsculas, de los señores, sus parientes y sirvientes, de los mandones menores, de los procesos de evangelización y bautismo, cambios de nombre propios, entre otros muchos más.

Los censos que se resguardan en México se refieren a los poblados de Quauchichinola, Huitzillan, Tepoztlán, Tepetenchic y Molotla, mientras que los fragmentos de Cracovia se refieren a Comoliuhcan y los de París a Colhuacan y Calnahuac, incluyendo todos otras parcialidades menores. Están escritos en náhuatl con grafías latinas

estos censos. Realizó un análisis pionero de una parte inicial del censo de Molotla en un trabajo que denomina "Casa y hacienda de un señor tlauica” y se refiere a los censos en sus estudios más generales; $\mathrm{cfr}$. Carrasco $(1972,1976 a, 1976 b)$. Ismael Díaz Cadena tradujo la parte de Tepoztlán (Díaz Cadena, 1978). También los censos de Molotla, Tepetenchic y Panchimalco ya fueron publicados en 1982 con una traducción al alemán por Hinz y sus colegas (cfr. Hinz et al., 1982). El censo de Quauchichinola fue publicado en 1993 por Sarah Cline con una traducción al inglés (cfr. Cline, 1993). Estos dos últimos autores publicaron estudios introductorios a los censos. Así, Hinz y sus colaboradores subrayan los temas de la crisis demográfica que se vivía en la década de 1540. También analizan la relación entre las tierras que ocupaba cada casa, el tributo y la estratificación social. Cline, en cambio, enfatiza el problema de la evangelización y del bautismo de la población indígena. Otros análisis etnohistóricos que se refieren a estos materiales han puesto de relieve el valor de estas fuentes para estudios demográficos o lingüísticos más detallados. Véase Lockhart, 1992; y Mc Caa, 1996. y ennumeran casa por casa a los habitantes de varios poblados, barrios y sub-barrios de la zona de Tepoztlán, Yautepec y Cuernavaca. Quisiera subrayar el hecho de que los documentos de Cracovia complementan a los que están en París, pues así puede ser más extensa la visión que se puede obtener de poblados menores que conforman los calpulli o los tlaxilacalli de poblados como Colhuacan, Calnahuac, Comoliuhcan y otros.

Las características de estos manuscritos son ligeramente distintas a las de los censos que están en México, como veremos en seguida. Los manuscritos Am. 3 y Am. 8 que pude consultar en la Biblioteca Jagiellowska muestran las mismas características físicas del manuscrito mex. 393 que se encuentra en París en la Biblioteca Nacional; y la lectura cuidadosa mostró que también en relación con el contenido ambos documentos se complementan perfectamente. Como se dijo, los dos están escritos, al parecer, en papel amate (ficus) y con tinta probablemente de huizache, aunque en Cracovia y en París no se han hecho estudios detallados del material con que fueron elaborados. Llama la atención el gran tamaño de las fojas y lo hermoso de las letras, que dan la impresión más de haber sido pintadas que escritas. Por cambios en la letra se puede ver que hubo varios amanuenses y que fueron los mismos en los fragmentos de París y de Cracovia. En ninguna parte de los dos fragmentos se menciona de manera explícita el lugar y la fecha en que fue elaborado el documento, aunque se dan pistas que en un futuro ayudarán a resolver el problema. ${ }^{5}$ Tanto el manuscrito Am. 3 de Cracovia que ya había sido encuadernado en Berlín y está en excelentes condiciones, como el Am. 8 que consta de dos fojas sueltas, así como el manuscrito mex. 393 de París (en fojas sueltas) conforman un censo de poblados contiguos. El censo completo debe haber sido un documento sumamente extenso de cientos de fojas, pues como dijimos, los fragmentos del Am. 3 y Am. 8 constan de un total de $75 \mathrm{y}$ dos fojas respectivamente escritas por los dos lados y el manuscrito mex. 393 de París contiene 32 fojas también escritas por ambos lados.

${ }^{5} \mathrm{Al}$ final del censo de Comoliuhcan se mencionan "las casas de Toribio en Tenantitla" y se dice que de ellas hicieron la cuenta Juan Ortiz y Martín de Peralta. 
En estos censos se registran, casa por casa, los habitantes de toda una región, anotándose el nombre del que preside la unidad doméstica, el nombre de su mujer y de sus hijos o los de sus parientes que viven en la misma casa, además de la cantidad de tierras de cultivo que posee y el tributo o contribución que paga. La característica que distingue a estos documentos de los que están en la Colección Antigua en la Biblioteca del Museo de Antropología e Historia de México, es el hecho de que no se da la información de la edad de cada uno de los hijos en cada casa, sino sólo la edad del hijo mayor, con lo que se puede deducir que los otros son menores. También es un rasgo particular el gran énfasis que se pone respecto a los funcionarios que se encargan de recoger el tributo de cada casa. Se da peculiar peso a la especificación de quién es el responsable de la recolección y entrega de las diferentes contribuciones que cada casa debe de dar. En cada casa de un sub-barrio o parcialidad menor se repite, por ejemplo, que es Alonso quien recoge el tributo y se lo da a Pablo quien finalmente hace la entrega al marqués (refiriéndose a Hernán Cortés): yn ytequihv quinechicova alo $\{n\}$ so quimaca pablo ahv $y\{n\}$ pablo quimaca malquex (Ms. Am. 3, f. 15v)

En contraste con el manuscrito mex. 393 de París, que presenta un problema complejo de ordenamiento por la foliación equivocada, ${ }^{6}$ el documento Am. 3 de Cracovia no presenta ese problema, con excepción de una foja última que no está en su lugar correcto. El manuscrito 393 que contiene el censo de lugares nombrados Calnavac y Colvacan, donde el funcionario o mandón de nombre Agustín recogía el tributo, menciona también parcialidades menores como Tlacochcalco y Olac. Explícitamente se denomina el primer barrio o parcialidad Tlapallan, después el "segundo barrio que tiene por nombre Tenantitla donde gobierna Miguel" o sea, yzca yconcalpuli ytocayoca Tenantitla onca tlapachova Miguel, el "tercer barrio de Ixtlahuaca" al decirse yzca yquecalpulli ytocayoca Yxtlavaca... y otros poblados vecinos. Con los documentos en Cracovia se completa el censo ya que en el

${ }^{6}$ Las fojas que están sin orden correcto en París se refieren sobre todo a Calnahuac, así como al barrio de Ixtlahuaca y de Olac.

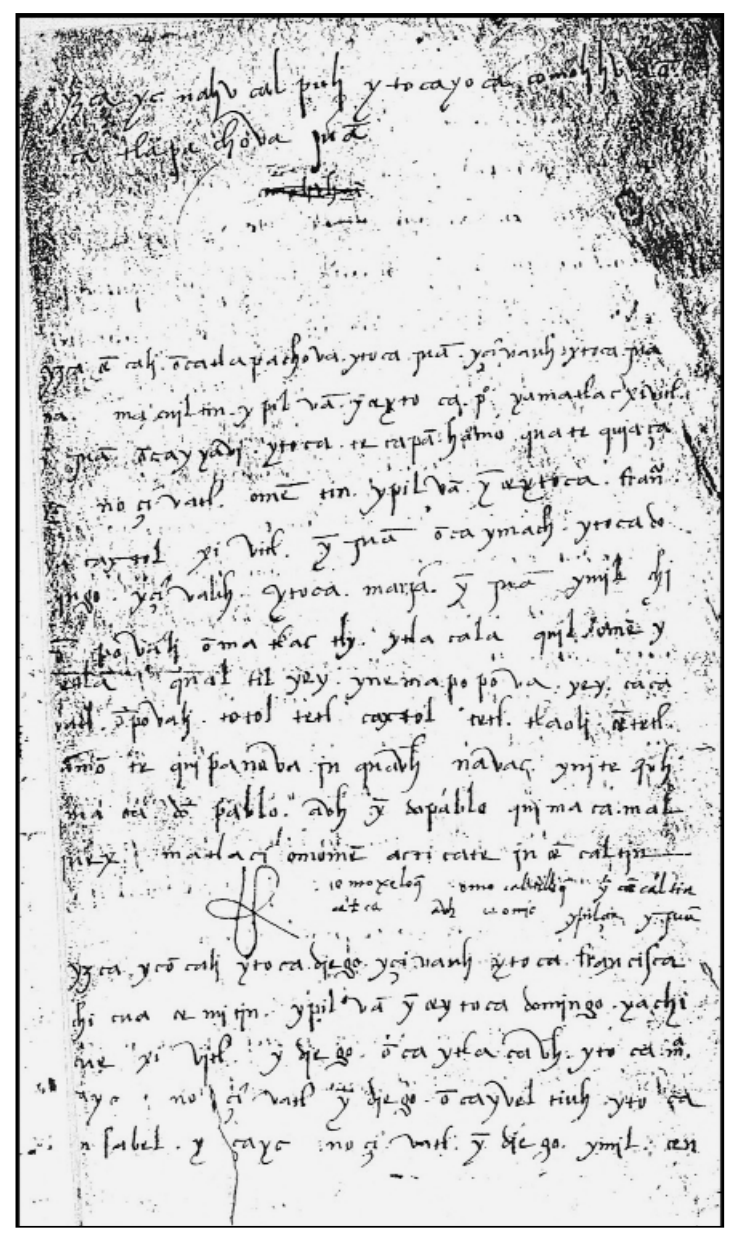

Códice Am.3 de Cracovia (detalle).

Am. 3 se tiene el cuarto barrio: $y z c a$ ycnahvcalpuli ytocayoca comolihucan onca tlapachova juan. El censo de esta parcialidad está completo y también se mencionan sus barrios menores como Nochtla, Tecpa/../co. En las pequeñas anotaciones al final de las casas, que se refieren a los movimientos de población, los destinos de los jóvenes que se casaron y se fueron, o los lugares de donde proceden hijas viudas o padres viudos que vuelven a integrarse a las casas de sus parientes, se mencionan los mismos lugares que en los otros censos, sobre todo Caltonco, Calitec, Ochpanco, Atenco, Tepetlapa, Tenantitla, Yxtlavacan, entre muchos otros. Es, pues, evidente que los manuscritos Am. 3 y Am. 8 de Cracovia comple- 
tan el fragmento del censo del 393 de la Biblioteca Nacional de Francia en París.

Hoy día es difícil localizar todas estas poblaciones mencionadas pues esos topónimos — muchos de origen xochimilca- se repiten con frecuencia y son muy comunes en el sur del valle de México durante el siglo XVI y en la región del actual estado de Morelos. Lo más probable es que se trate de poblados cercanos a Cuernavaca ubicados al sur y este de la actual capital del estado de Morelos, en los valles de Jiutepec y los conlindantes de Tepoztlán y Yautepec. La mayoría de esos parajes quedaron abandonados durante el siglo XVI cuando muchos poblados desaparecieron o perdieron su nombre numerosas demarcaciones en tanto que asentamientos separados. Ante la crisis demográfica ocurrida en las décadas de 1540 y 1570 una gran cantidad de lugares quedaron despoblados.

Además, importantes transformaciones ocurrieron a partir de las congregaciones que en el siglo XVI, y sobre todo a fines de ese siglo y a inicios del XVII, obligaron a aquellos habitantes de la zona que habían logrado sobrevivir ante las epidemias a reubicarse y migrar a determinados pueblos que son los que, por lo general, han perdurado hasta la actualidad. A partir del siglo XVII se dio también una gran cantidad de mercedes de tierras a nuevos protagonistas que fundaron en esta región empresas ganaderas, cerealeras y azucareras, es decir, ranchos y haciendas. Por esta razón desaparecieron las antiguas denominaciones de parajes, barrios y parcialidades o quedaron incorporadas en esas nuevas entidades. ${ }^{7}$

Los dos fragmentos de censo indígena que analizamos en Cracovia revelan una práctica antigua de llevar padrones de la población y cuentas rigurosas de sus tributos en la región que hoy conforma el estado de Morelos. Como la entrega de contribuciones o impuestos que debían hacer los agricultores estaba relacionada con la cantidad de tierra que cada jefe de familia había obtenido por asignación o herencia, toda casa y familia debía estar empadronada. Así, estas largas listas se elaboraban para llevar cuenta exacta de las tierras disponibles y de los tri-

\footnotetext{
${ }^{7}$ Mentz, 1988, 1997
}

butos que debía pagar cada pequeña parcialidad, barrio mayor, poblado y ciudad.

Hay que tener presente que las mismas enumeraciones de casas, sus moradores, tierras y tributos se registraron también en otro tipo de documentos del siglo $\mathrm{XVI}$, como las fuentes pictográficas; en especial, por ejemplo, el Códice Vergara, el Santa María Asunción y el Otlazpan. Esos documentos son los que mayor semejanza tienen con el texto de nuestros censos, además de otros como el Memorial de Tepetlaostoc y el llamado de Tepoztlán ubicado en Tulane. ${ }^{8}$ Todos ellos hacen alusión a la forma precisa de pago de tributo de ciertos individuos o a la cuenta detallada de los habitantes de los poblados y dan razón de la existencia de esta práctica de llevar censos rigurosos en escritura pictográfica indígena probablemente desde época anterior a la conquista.

Como hemos mencionado, los datos contenidos en estos padrones se corregían conforme era necesario, por ejemplo, cuando nacían hijos, moría algún miembro de la familia, cuando se mudaba una familia a otra parte y dejaba sus tierras o cuando llegaban nuevos inmigrantes de otras parcialidades. Es probable que censos como éstos fueran revisados cada dos o tres años, anotándose cómo se habían modificado las familias por muerte o por nacimientos de nuevos miembros.

Una peculiaridad estilísitica que se observa en todos los fragmentos de estos documentos en grafías latinas y en náhuatl es que cada casa inicia con una pequeña marca diagonal del lado izquierdo que muestra el inicio de una nueva casa censada. En unos fragmentos, esta pequeña diagonal está más elaborada — por ejemplo, en los que custodia el INAH - y en otros menos. En el manuscrito mex. 393 es una pequeña raya solamente, al igual que en el Am. 3 y Am. 8 de Cracovia.

Para la historia social del estado de Morelos es de gran interés el tipo de información sobre cada unidad doméstica que se da en estos manuscritos. Los datos que se anotan difieren un poco entre censo y censo. En los de París y de Cracovia se expresa el número y parentesco de per-

${ }^{8}$ Cfr. Williams, 1984, 1988; Harvey, 1986; Leander, 1967; Valle, 1993; Brotherston, 1999. 
sonas que conforman cada casa o "familia" en el sentido romano, es decir, a todos los que viven bajo el mismo techo en dependencia del pater familiae, incluyendo a sus parientes y a todos los sirvientes, es decir, incluyendo al famulus. En los censos se anota en primer lugar el nombre del que preside la unidad doméstica y de su mujer, sus hijos — añadiendo la edad del hijo mayor — más los nombres de las demás personas que viven en dicha unidad. Después se informa sobre las tierras de cultivo de que dispone esta unidad doméstica y la contribución o tributo que le corresponde, tanto de la contribución llamada tlacalaquilli (lo que se encierra en la casa, que abarca ciertas mantas), el llamado tetlacualtilli (que en traducción literal sería "comida para la gente", pero se refiere a otros textiles o mantas) y la entrega de textiles de mano menores llamados nemapopovayaloni, así como, al último, cacao, huevos, maíz desgranado y servicios que se debían hacer en Cuernavaca. Después se da el nombre del mandón que recoje en dicha casa esas contribuciones y descripción exacta de si él mismo se las lleva al marqués o si las da a su vez a otro funcionario superior quien finalmente se las entrega. Como ya se mencionó, también al final se informa sobre los cambios ocurridos en el número de miembros que conforman la familia, ya sea por haberse incrementado gracias al nacimiento de hijos, a la llegada de parientes o por haber disminuido ante la salida de hijos que se casaron o mudaron a otro lugar. Esos comentarios que siempre aparecen en letra más pequeña dan dinamismo temporal al registro censal, pues se pueden observar los cambios ocurridos en la vida de algunos integrantes de las unidades domésticas, algunas prácticas reiteradas y sus vínculos con otras parcialidades a donde se mudaron, entre otros.

Los manuscritos de Cracovia y de París difieren de los de Molotla, Tepetenchic, Huizillan y Quauchichinola del INAH al tratarse de censos de poblados menores, de escasa importancia en la jerarquía política. Quauchichinolla y Huizillan, por ejemplo, eran considerados un altepetl (altepeme en plural) y el título de los señores de esos reinos o pequeños señoríos era el de tlatoani, a los señores de Molotla y de Tepetenchic se les otorga el título de tecuhtli en esos censos bajo custodia del INAH. En cambio, las parcialidades registradas en los documentos Am. 3 y 8 de Cracovia y Ms. mex. 393 de la BNF tienen por autoridad un funcionario al que no se le da título o cargo expreso sino que se menciona simplemente como "el que gobierna aquí se llama...” (onca tlapachoa in itoca...). Por lo tanto, resultan evidentes diferencias sociales, por ejemplo, entre el funcionario llamado Agustín de Calnahuac, quien recoge al parecer los tributos de toda esa zona, y el tecuhtli Martín Molotecatl. Agustín evidentemente es un funcionario de menor categoría que Martín Moletecatl, el señor de Molotla, pues cuenta con menos tierras y menos dependientes.

En el caso de Colhuacan y Calnahuac (Ms. mex. 393) también resalta el papel de un "don Pablo" y en el caso de Comoliuhcan (Ms. Am. 3) de don Diego Xolotecatl. ${ }^{9}$ La casa de este señor está al inicio del censo de este barrio. Cuenta con 140 unidades de tierra (mientras lo normal son 20 o incluso menos en esta zona, según los datos de las otras casas) lo que explica su importancia económica y política en esa enmarcación. Además, es quien funje como recaudador en Comoliuhcan. En el mencionado caso de Agustín, el mandón que recoje los tributos en Colhuacan y sus barrios (Ms. BNF mex. 393) tiene una jerarquía menor, lo que se refleja en el hecho de que él mismo controla sólo 65 unidades de tierra. A la vez, también hay recogedores todavía menores que son los que viven siempre en las primeras casas al inicio de la parcialidad que presiden.

Como en casi todas las listas, al final se mencionan los leñadores o los que se encargan de cuidar flores, pues ellos por lo general no pagan las mismas contribuciones en textiles como los demás.

Como hemos subrayado, en los manuscritos de Cracovia y de París no tenemos las cuentas finales que abarcan a toda la población del pequeño reino o altepetl al que pertenecían Calnahuac y Colhuacan. Sin embargo, es evidente que en el caso de los habitantes de estos lugares se trata de comuneros más pobres, pues sus sementeras son

\footnotetext{
${ }^{9}$ En comparación con los señores de otros poblados mencionados, como Martín Molotecatl, tecuhtli de Molotla, también resulta Diego Xolotécatl de menor rango. Es obvio, en general, que los poblados de los censos en París o Cracovia son pequeños y carecen de la importancia o rango de los mencionados en los censos de Quauchichinola, Huitzilan o Molotla.
} 
casi todas iguales que su tributo. Tienen parcelas del tamaño de 20 unidades en promedio y la entrega sistemática de una manta de tlacalaquilli completa al año, más tres mantas de tetlacualtilli y tres servilletas. Además, entregaban cacao, huevos, maíz desgranado y acudían a la cabecera Cuauhnahuac a servir. La composición de las familias era por lo general de una o dos parejas casadas. De manera indistinta quedaba a veces el hijo casado por algún tiempo en su casa paterna hasta que se le daban tierras, o se iba a casar a otro lado, donde vivía en casa de la nueva esposa, hasta que se independizaba se le otorgaban tierras y fundaba casa propia. Muy común era que hermanos menores vivieran en casa de un hermano mayor o que sobrinos solteros o casados vinieran a vivir con el tío.

El resultado del promedio de personas por casa en los documentos de Cracovia y París es sorprendente, pues es mucho menor al reportado por los otros altepeme. Así, gracias a que las poblaciones censadas en estos textos son tan diferentes a las estudiadas hasta ahora, podemos contrastar también el tipo de familias que viven en estos lugares, su diferencial acceso a la tierra y también registrar la diferencia entre los barrios más grandes y los pequeños.

Sorprende la gran variedad de número de personas registradas en cada casa, siendo en unas el promedio mayor a 5.5 o 6.4 en Tlacochcalco y Tlapallan, respectivamente; y en otras de sólo 4.2 o 3.8, en los barrios de Calnahuac y Tepetenchic-Tenantitla. Esto contrasta con los estudios que ya se han elaborado de las casas del señor de Molotla y de sus dependientes (con promedio de 8.3 por casa) y también con los resultados presentados por Hinz y sus colegas, quienes muestran que en promedio vivían en Tepetenchic 7.0 personas en cada unidad doméstica.

Para la historia demográfica estos resultados tan detallados son de importancia, pues existen muy escasas evidencias históricas que sean más exactas para el cálculo de la densidad demográfica, y por ello los historiadores y también los arqueólogos se ven precisados a aventurar ciertas cifras como aproximaciones. Para calcular la población de la cercana ciudad de Xochicalco en el epiclásico, por ejemplo, el arqueólogo Hirth propone multiplicar ya sea por cinco o por siete para cada casa; de la misma manera, los historiadores coloniales sugieren diversas multiplicaciones para el cómputo de la población a partir del número de tributarios o simplemente multiplican por cinco para calcular el número de familiares que tiene cada casa indígena para el periodo virreinal. ${ }^{10}$ Con estos censos de aproximadamente 1540 podemos mostrar que para esta zona hay que diferenciar los poblados internamente y que para ciertos lugares donde vivía la elite y sus parientes y allegados pueden ser correctas cifras cercanas a siete, y en cambio, para otras zonas sería más correcto multiplicar por tres o cuatro.

Podemos finalizar reiterando la importancia que tienen estas fuentes tan tempranas en lengua náhuatl dispersas entre bibliotecas de México, París y Cracovia para la historia social de México, tanto en relación con el pasado prehispánico como el colonial. Con el análisis de este tipo de documentos se puede enriquecer mucho la discusión sobre fonología, gramática y terminología náhuatl, sobre formas de asentamientos indígenas anteriores a la llegada de los europeos, sobre el tamaño de las unidades domésticas, el parentesco y la movilidad espacial de los miembros de las distintas familias, sobre la forma de distribuir las tierras y las contribuciones entre la población rural, sobre la estratificación política y social y el ordenamiento espacial, entre muchas otras cuestiones.

Así, documentos alojados en repositorios tan lejanos como los de la Biblioteca Jagiellowska en Cracovia pueden ayudar a obtener datos concretos que arrojan mucha luz sobre estos temas de historia social, demografía y lingüistica náhuatl.

\section{Bibliografía}

Bankmann, Ulf, 1970, "Manuscripta Americana der Staatsbibliothek in Berlin", en Verhandlungen des XXXVIII Internationales Amerikanistenkongresses, Stuttgart-Muenchen, 12-18 de agosto, 1968, t. II, Muenchen.

${ }^{10}$ Gebhard, 1962; Hirth, K., 2000, vol. 1, pp. 138ss. Para una crítica reciente de estas prácticas de cálculos de demografía histórica véase Rabell Romero, 2001. 
Broda, Johanna y Pedro Carrasco (eds.), 1976, Estratificación social en la Mesoamérica prehispánica, SEP-INAH, México.

Carrasco, Pedro, 1972, "La casa y la hacienda de un señor Tlalhuica”, en Estudios de Cultura Náhuatl, vol. X.

— 1976 a, "Estratificación social indígena en Morelos durante el siglo XVI", en Broda y Carrasco.

— 1976b, "The Joint Family in Ancient Mexico: The Case of Molotla", en Hugo Nutini et al. (eds.), Essays on Mexican Kinship, University of Pittsburgh Press, Pittsburgh.

__ 1989, "Los mayeques", en Historia Mexicana, vol. 34, núm. 1.

Brotherston, Gordon, 1999, El Códice de Tepoztlán, imagen de un pueblo resistente, Editorial Pacífica, San Francisco, Estados Unidos.

Castillo, Víctor, 1984, Estructura económica de la sociedad mexica según las fuentes documentales, UNAM, México.

Cline, Sarah, 1978, The Book of Tributes. Early Sixteenth-Century Náhuatl Censuses from Morelos, Latin American Center Publications, Universty of California, Los Ángeles.

Díaz Cadena, Ismael, 1978, Libro de los tributos del marquesado del Valle, INAH, México.

Durán, fray Diego, 1984, Historia de las Indias de Nueva España e islas de la tierra firme, 2 vols., Porrúa, México.

Gerhard, Peter, 1962, México en 1742, Editorial José Porrúa e hijos, México.

Harvey, H.R., 1986, "Household and Family Structure in Early Colonial Tepetlaoztoc: An Analysis of the Códice Santa María Asunción", en Estudios de Cultura Náhuatl, núm. 18, p. 275-294.

Harvey y Prem (comps.), 1984, Explorations in Ethnohistory, University of New Mexico Press, Albuquerque.

Hicks, Fredric, 1974, "Dependent Labor in Prehispanic Mexico", en Estudios de Cultura Náhuatl, vol. 11.

__ 1976, "Mayeque y Calpuleque en el sistema de clases del México antiguo", en Broda y Carrasco.

_ , "Rotational Labor and Urban Development in Prehispanic Tetzcoco" en Harvey y Prem (comps.), Explorations in Ethnohistory, University of New Mexico Press, Albuquerque.

Hinz, Eike et al., 1983, Aztekischer Zensus. Zur indianischen Wirtschaft und Gesellschaft im Marquesado um 1540, 2 vols., Verlag fuer Ethnologie, Hannover, Alemania.
Hirth, Kenneth, 2000, Ancient Urbanism at Xochicalco. The Evolution and Organization of a Prehispanic Society, vol. 1, The University of Utah Press, Salt Lake City.

Leander, Birgitta, 1967, Códice de Otlazpan, 2 vols., INAH, México.

Lockhart, James, 1992, The Nahuas After the Conquest. A Social and Cultural History of the Indians of Central Mexico, Sixteenth Through Eighteenth Centuries, Stanford University Press (versión en castellano publicada en FCE, 1999).

Macías, Marcos, 1989, "La antropometría indígena en las medidas de longitud (en documentos de la ciudad de México del siglo XVI)", en Primer Coloquio de Documentos Pictográficos de Tradición Náhuatl, UNAM, México, p. 177-209.

Martínez, Hildeberto, 1984, Tepeaca en el siglo XVI. Tenencia de la tierra y organización de un señorío, CIESAS, Ediciones de la Casa Chata, México.

Mentz, Brígida von, 1988, Pueblos de indios, mulatos y mestizos. Los campesinos y la protoindustrialización en Morelos, CIESAS, México, 1988.

_ et al., 1997, Haciendas de Morelos, M.A. Porrúa, Gobierno del Estado de Morelos, México.

Mohar, Luz María, 1987, El tributo mexica en el siglo XVI, CIS-INAH, México.

_ 1990, La escritura en el México Antiguo, 2 vols., Plaza y Valdés, México.

Ramírez Montes, Mina, 1990, Manuscritos novohispanos. Ejercicios de lectura, UNAM, México.

Rabell Romero, Cecilia Andrea, 2001, Oaxaca en el siglo XVIII: Población, familia y economía, tesis de doctorado, Centro de Estudios Demográficos y de Desarrollo Urbano, El Colegio de México, México.

Valle P., Perla, 1993, Memorial de los indios de Tepetlaóztoc o Códice Kingsborough, INAH, col. Científica, México.

Williams, Barbara, 1984, "Mexican Pictorial Cadastral Registers”, en Harvey y Prem (comps.), pp. 103-125. y H. R. Harvey, 1988, "Content, Provenience and Significance of the Codex Vergara and the Códice de Santa María Asunción", en American Antiquity, núm. 53, pp. 337-351.

Zavala, Silvio, 1984, Tributos y servicios personales de indios para Hernán Cortés y su familia. Extractos de documentos del siglo XVI, AGN, México. 